\title{
2011 Engineering Salaries: AAES Industry Survey Shows Where the Money Is
}

\section{Joann Cantrell}

Despite a fragile U.S. economy slowly making its way toward a recovery, engineers seemingly have been able to fend off the negative effects of wage freezes. To fully understand how much engineers are paid, it's important to know that there are well over a dozen engineering specialties, each with various earnings, so the salary of an engineer depends on the specialization.

The 2011 survey of engineers' compensation, conducted by the Engineering Workforce Commission (EWC) of the American Association of Engineering Societies (AAES), collected salaries of engineers based on discipline, levels of experience, supervisory responsibility, academic preparation and location. Other classifications in the survey included industry sectors and size of employers.

The data reflects salaries as of February 1,2011 and the number of engineers represented in the survey is 39,907 . The results revealed that the average salary for engineers in all industries, at all degree levels, with five years of experience or less was $\$ 72,705$. At the other end of the occupational spectrum, engineers with more than 35 years of work experience earned a median annual salary of $\$ 103,397$. For specifics, see Table I for salaries by discipline; Table II for salaries based on experience, supervisory responsibility, and level of education; and Table III for salaries based on experience and employer

For more than 40 years, the EWC has studied to let engineers see how their salaries stack up against their peers. To review the full report, visit the AAES website at www.aaes.org/ewc/salary.

Joann Cantrell is a freelance writer.

\begin{tabular}{lccccccc}
\hline \multicolumn{7}{c}{ Table I. Median Annual Salaries for Engineers by Discipline (in Dollars) } \\
\hline & \multicolumn{7}{c}{ Number of Years after B.S. } \\
\cline { 2 - 8 } Discipline & $\mathbf{0}$ & $\mathbf{5}$ & $\mathbf{9 - 1 0}$ & $\mathbf{1 3 - 1 6}$ & $\mathbf{2 1 - 2 5}$ & $\mathbf{3 5 +}$ \\
\hline Aerospace Engineers & - & - & $\mathbf{9 2 , 9 2 9}$ & $\mathbf{1 0 2 , 7 2 4}$ & $\mathbf{1 0 8 , 3 6 1}$ & $\mathbf{1 1 4 , 6 0 8}$ \\
Computer Engineers & - & 69,158 & 82,693 & 92,263 & 98,333 & 108,981 \\
Chemical Engineers & - & 68,205 & 85,806 & 90,808 & 101,686 & 116,430 \\
Civil Engineers & 53,331 & 59,036 & 67,468 & 78,099 & 86,440 & 86,598 \\
Electrical Engineers & 62,321 & 73,117 & 83,873 & 93,521 & 103,824 & 104,104 \\
Environmental Engineers & 51,002 & 59,675 & 66,237 & 81,684 & 100,579 & 110,506 \\
General Engineers & - & 78,977 & 94,218 & 97,935 & 98,182 & 100,914 \\
Industrial Engineers & - & 71,504 & 85,931 & 92,098 & 104,273 & 106,369 \\
Materials Engineers & - & - & 83,634 & 96,285 & 114,029 & 130,586 \\
Mechanical Engineers & 56,208 & 74,225 & 86,945 & 97,512 & 108,125 & 120,806 \\
Nuclear Engineers & - & - & - & 96,017 & 103,130 & 145,396 \\
\hline
\end{tabular}

Table II. Median Annual Salaries for Engineers Based on Experience, Supervisory Responsibility, and Level of Education (in Dollars)

\begin{tabular}{|c|c|c|c|c|c|c|}
\hline \multirow[b]{2}{*}{ Employer } & \multicolumn{6}{|c|}{ Number of Years after B.S. } \\
\hline & 0 & 5 & $9-10$ & $13-16$ & $21-25$ & $35+$ \\
\hline \multicolumn{7}{|c|}{$\overline{\text { Nonsupervisory }}$} \\
\hline B.S. & 58,354 & 70,278 & 77,596 & 84,959 & 92,262 & 92,000 \\
\hline M.S. & - & 80,504 & 90,605 & 96,710 & 99,987 & 100,338 \\
\hline Ph.D. & - & - & 96,102 & 101,523 & 110,967 & 130,568 \\
\hline \multicolumn{7}{|c|}{ Supervisory } \\
\hline B.S. & - & - & 77,364 & 89,536 & 100,379 & 109,424 \\
\hline M.S. & - & 118,894 & 122,663 & 125,265 & 129,657 & 124,652 \\
\hline Ph.D. & - & - & - & 120,836 & 139,164 & 157,000 \\
\hline
\end{tabular}

Table III. Median Annual Salaries for Engineers Based on Experience and Employer (in Dollars)

Employer

Architecture, Engineering, and Related Services

Primary Metal Manufacturing

Fabricated Metal Product

Manufacturing

Public Administration

Motor Vehicle

Manufacturing

Professional, Scientific, and

Technical Services

R\&D Organizations

Utilities

Electric Power Generation,

Transmission \& Distribution

Mining

All Nonmanufacturing

All Manufacturing

\begin{tabular}{|c|c|c|c|c|c|}
\hline \multicolumn{6}{|c|}{ Number of Years after B.S. } \\
\hline 0 & 5 & $9-10$ & $13-16$ & $21-25$ & $35+$ \\
\hline 54,080 & 61,630 & 75,499 & 89,088 & 97,436 & 103,641 \\
\hline- & - & - & 85,507 & 89,714 & 105,370 \\
\hline - & - & 71,184 & 76,924 & 85,219 & 85,728 \\
\hline 53,164 & 67,775 & 71,303 & 81,733 & 92,643 & 94,553 \\
\hline 62,321 & 78,957 & 92,471 & 102,160 & 114,645 & 122,617 \\
\hline 52,000 & 65,243 & 80,366 & 90,907 & 100,056 & 116,251 \\
\hline- & 70,677 & 82,751 & 92,418 & 101,000 & 125,000 \\
\hline 61,112 & 67,932 & 65,518 & 76,754 & 94,604 & 87,798 \\
\hline 62,678 & 72,723 & 71,922 & 83,584 & 105,418 & 122,828 \\
\hline- & 71,358 & 80,416 & 91,426 & 107,772 & 118,724 \\
\hline 52,475 & 66,892 & 77,907 & 86,979 & 95,287 & 105,742 \\
\hline 64,512 & 77,869 & 89,455 & 98,956 & 106,491 & 103,450 \\
\hline
\end{tabular}

